\title{
Computational Dynamics of Arterial Blood Flow in the Presence of Magnetic Field and Thermal Radiation Therapy
}

\author{
T. Chinyoka ${ }^{1}$ and O. D. Makinde ${ }^{2}$ \\ ${ }^{1}$ Center for Research in Computational and Applied Mechanics, University of Cape Town, Rondebosch 7701, South Africa \\ ${ }^{2}$ Faculty of Military Science, Stellenbosch University, Private Bag X2, Saldanha 7395, South Africa \\ Correspondence should be addressed to T. Chinyoka; tchinyok@vt.edu
}

Received 24 March 2014; Accepted 31 March 2014; Published 22 April 2014

Academic Editor: Raseelo Joel Moitsheki

Copyright ( 2014 T. Chinyoka and O. D. Makinde. This is an open access article distributed under the Creative Commons Attribution License, which permits unrestricted use, distribution, and reproduction in any medium, provided the original work is properly cited.

\begin{abstract}
We conduct a numerical study to determine the influence of magnetic field and thermal radiation on both velocity and temperature distributions in a single blood vessel. The model here assumes that blood is a Newtonian incompressible conducting fluid with radially varying viscosity due to hematocrit variation. The transient equations of momentum and energy transport governing the flow in an axisymmetric configuration are solved numerically using a semi-implicit finite difference method. Results are presented graphically and discussed both qualitatively and quantitatively from the physiological point of view. The results of this work may enhance current understanding of the factors that determine the effects of hyperthermia treatment on tumor tissues.
\end{abstract}

\section{Introduction}

Blood flow in a large blood vessel has a profound influence on the efficiency of thermal therapy treatment [1-3]. In pathological situations, thermal radiation therapy is one of the treatments employed by medical practitioners [4-6]. The procedure involves transmitting heat below the skin surface into tissues and muscles. Deep heat speeds up healing by increasing blood flow to the injury. Electromagnetic heat, such as shortwaves and microwaves, sends heat up to 2 inches into the tissue and muscles. It works best for injuries in joints, muscles, and tendons. Heat therapy may help reduce pain. Moreover, hyperthermia treatment has been demonstrated as effective during cancer therapy in recent years. Its objective is to raise the temperature of pathological tissues above cytotoxic temperatures $\left(41-45^{\circ} \mathrm{C}\right)$ without overexposing healthy tissues $[7,8]$. Temperature distribution within tissues primarily depends on tissue thermal conductivity, the heating source's power deposition pattern characteristics, and heat transfer resulting from blood flow [9-11]. An important source of temperature nonuniformity is the presence of large vessels entering the heated volume and carrying blood at a lower systemic temperature $\left(37^{\circ} \mathrm{C}\right)$. The design of delivered power devices and numerous theoretical, experimental, and clinical studies have demonstrated that large blood vessels may produce localized cooling regions within heated tissues during hyperthermia treatment $[12,13]$. Kolios et al. [14] demonstrated the effect of large blood vessel in heated tissues and showed that the dissipation of heat from heated tissues was carried out by convection through blood flow and also by conduction process. A numerical study on the impact of large vessels on the temperature uniformity during hyperthermia treatment assuming steady-state condition was conducted by Creeze and Lagendijk [15]. They reported that the presence of a large vessel may result in nonuniform temperature resulting in possible underdosage. Cho and Hyun [16] assumed a sinusoidal variation of the velocity at the pipe inlet in a numerical study of pulsatile flow and heat transfer characteristics within a pipe. Seo Young Kim et al. [17] analyzed numerically the heat transfer characteristics of fully developed pulsatile flow in a channel, assuming a sinusoidal variation of the velocity at the inlet of the channel. In recent time, several authors have also investigated the flow structure and heat transfer characteristics of Newtonian and non-Newtonian fluid models in order to understand the physiological flow systems [18-21]. However, in the large 


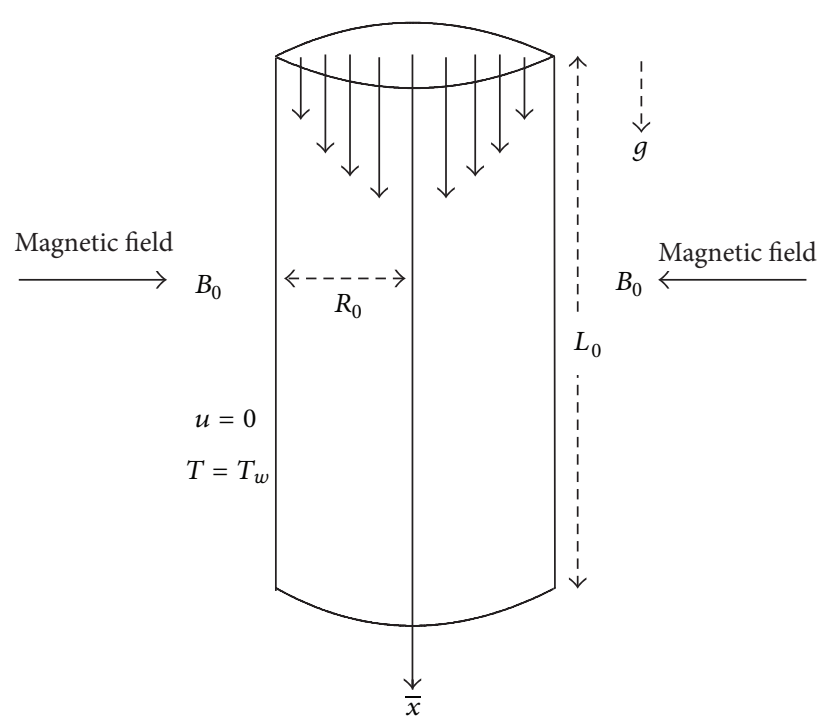

FIGURE 1: Geometry of the problem.

blood vessel it is a good approximation to consider whole blood as a Newtonian viscous fluid [22].

So far, the theoretical studies dealing with the combined effects of thermal radiation absorption and magnetic field on the transient flow of blood in a large artery with viscous dissipation have received very little attention. It was observed that the heart rate decreases by exposing biological systems to an external magnetic field. The electrocardiography (ECG) pattern taken in the presence of a magnetic field not only provides information on blood flow but also offers a new noninvasive method of studying the cardiac performance [23]. Moreover, the red blood cell (RBC) is a major biomagnetic substance, and the blood flow may be influenced by the magnetic field. The magnetic properties of RBCs play important roles in the increase in blood viscosity during exposure to a static magnetic field. Motivated by the above studies, our aim is to investigate the combined effects of thermal radiation and magnetic field on transient flow of blood in a large blood vessel. In Section 2 the mathematical formulation of the problem is established. The numerical solutions to the problem are obtained in Section 3. Qualitative and quantitative discussions of the solutions are given in Section 4.

\section{Formulation of the Problem}

Consider unsteady, axisymmetric incompressible Newtonian blood flow through a rigid vessel of length $L$ and radius $R_{0}$. The geometry of the problem and the coordinate system are shown in Figure 1.

For the development of mathematical model, the following assumptions are made.

(i) The flow is incompressible so that the simple equation of state for a Boussinesq fluid holds in gravitational field acting in reverse direction to $y$. (ii) Viscosity of blood varies radially with maximum magnitude along the arterial centerline due to the accumulation of red blood cells (RBC) in this region.

(iii) The temperature difference of the blood and artery is high enough for radiative heat transfer to be valid.

(iv) Magnetic field applied externally is of constant strength.

(v) The electromagnetic force produced and the electrical conductivity are very small.

(vi) The flow through the intimal and media layers and the deformation of blood vessels are neglected.

Under the above mentioned assumption, the one-dimensional equations of motion and energy balance for the unsteady and axisymmetric flow of blood through an artery provided are [22-25]

$$
\begin{gathered}
\rho \frac{\partial u}{\partial t}=\frac{\partial \bar{P}}{\partial \bar{x}}+\frac{1}{r} \frac{\partial}{\partial r}\left(r \bar{\mu} \frac{\partial u}{\partial r}\right)-\sigma_{e} B_{0}^{2} u+\rho g \beta\left(T-T_{0}\right) \\
\rho c_{p} \frac{\partial T}{\partial \bar{t}}=k \frac{1}{r} \frac{\partial}{\partial r}\left(r \frac{\partial T}{\partial r}\right)-\frac{\partial q_{r}}{\partial r}+\bar{\mu}\left(\frac{\partial u}{\partial r}\right)^{2} \\
u(r, 0)=1-r^{2}, \quad T(r, 0)=0 \\
\frac{\partial u}{\partial r}(0, \bar{t})=\frac{\partial T}{\partial r}(0, \bar{t})=0, \quad \text { for } \bar{t}>0 \\
u(1, \bar{t})=0, \quad T(1, \bar{t})=T_{w}, \quad \text { for } \bar{t}>0
\end{gathered}
$$

where $u$ is the blood axial velocity, $T$ is the blood temperature, $T_{w}$ is the arterial wall temperature, $T_{0}$ is the blood reference temperature, $g$ is the gravitational acceleration, $\rho$ is the density, $\beta$ is the thermal expansion coefficient, $B_{0}$ is the intensity of magnetization, $\sigma_{e}$ is the electric conductivity of the blood, $R_{0}$ is the radius of the normal blood vessel, $k$ is the blood thermal conductivity, and $\bar{P}$ is the blood pressure. The 
most general differential approximation for the radiative flux $q_{r}$ as given by Cheng [26] is

$$
\frac{d}{d r}\left(\frac{1}{\alpha} \frac{d q_{r}}{d r}\right)-3 \gamma^{3} q_{r}-16 \gamma \sigma T^{3} \frac{d T}{d r}
$$

where $\gamma$ is the absorption coefficient and $\sigma$ is the StefanBoltzman constant. For the optically thin case that we adopt in the present study $\gamma \ll 1$ and an approximate form of the radiative flux equation could be taken as $[1,2,26]$

$$
\frac{d q_{r}}{d r}=4 \gamma \sigma\left(T_{0}^{4}-T^{4}\right)
$$

Following Lih [27], the transverse variation in the blood viscosity $\mu(r)$ is taken as

$$
\bar{\mu}(r)=\mu_{0}[1+\operatorname{ch}(r)]
$$

where $c=2.5$ (a constant), and $h(r)$ is the hematocrit function given as

$$
h(r)=h_{m}\left[1-\left(\frac{r}{R_{0}}\right)^{n}\right]
$$

where $h_{m}$ is the maximum hematocrit at the center of the blood vessel and $n(\geq 2)$ is a parameter determining the exact shape of the profile. Equation (5) is valid only for dilute suspension of red cells which are assumed spherical $[14,15]$. We introduce the following dimensionless quantities:

$$
\begin{aligned}
& y=\frac{r}{R_{0}}, \quad w=\frac{u}{U}, \quad \theta=\frac{T-T_{0}}{T_{w}-T_{0}}, \\
& \mathrm{Gr}=\frac{\rho g \beta\left(T_{w}-T_{0}\right) R_{0}^{2}}{U \mu_{0}}, \quad \mathrm{Br}=\frac{\mu_{0} U^{2}}{k\left(T_{w}-T_{0}\right)}, \\
& \operatorname{Re}=\frac{\rho U R_{0}}{\mu_{0}}, \quad M=\frac{\sigma_{e} B_{0}^{2} R_{0}^{2}}{\mu_{0}}, \\
& \mathrm{Ra}=\frac{4 \sigma \gamma R_{0}^{2}\left(T_{w}-T_{0}\right)^{3}}{k}, \quad \operatorname{Pr}=\frac{\mu_{0} c_{p}}{k}, \quad \mu=\frac{\bar{\mu}}{\mu_{0}}, \\
& b=c h_{m}, \quad x=\frac{\bar{x}}{R_{0}}, \quad P=\frac{\bar{P} R_{0}}{U \mu_{0}}, \quad L=-\frac{\partial P}{\partial x}, \\
& h=\frac{T_{0}}{T_{w}-T_{0}}, \quad a=1+b, \quad t=\frac{U \bar{t}}{R_{0}} .
\end{aligned}
$$

Substituting (5) into (1), we obtain the following dimensionless governing equations:

$$
\begin{aligned}
& \operatorname{Re} \frac{\partial w}{\partial t}=\frac{1}{y} \frac{\partial}{\partial y}\left[y\left(a-b y^{n}\right) \frac{\partial w}{\partial y}\right]-M w+L+\operatorname{Gr} \theta \\
& \operatorname{Re} \operatorname{Pr} \frac{\partial \theta}{\partial t}=\frac{1}{y} \frac{\partial}{\partial y}\left(y \frac{\partial \theta}{\partial y}\right)+\operatorname{Br}\left(a-b y^{n}\right)\left(\frac{\partial w}{\partial y}\right)^{2} \\
& +\operatorname{Ra}\left[(\theta+h)^{4}-h^{4}\right], \\
& w(y, 0)=m\left(1-y^{2}\right), \quad \theta(y, 0)=0, \\
& \frac{\partial w}{\partial y}(0, t)=\frac{\partial \theta}{\partial y}(0, t)=0, \quad \text { for } t>0, \\
& w(1, t)=0, \quad \theta(1, t)=1, \quad \text { for } t>0,
\end{aligned}
$$

where $\mathrm{Ra}$ is the radiation absorption parameter, $\mathrm{Gr}$ is the Grashof number, $h$ is the temperature difference parameter, $L$ is the axial pressure gradient parameter, $M$ is the magnetic field parameter, $\mathrm{Br}$ is the Brinkman number, $\mathrm{Re}$ is the Reynolds number, $\operatorname{Pr}$ is the Prandtl number, $a, b$ are hematocrit constants, and $n$ is the hematocrit shape parameter. The volumetric flow $Q$ of blood in the artery is given by

$$
Q=2 \pi R_{0} \int_{0}^{1} w(y) y d y
$$

The dimensionless shear stress $\left(C_{f}\right)$ and the rate of heat transfer $(\mathrm{Nu})$ at the arterial wall are given by

$$
C_{f}=-\left.\frac{d w}{d y}\right|_{y=1}, \quad \mathrm{Nu}=-\left.\frac{d \theta}{d y}\right|_{y=1} .
$$

In the following section, (7) are solved numerically and the skin-friction together with the arterial wall heat transfer rate is computed.

\section{Numerical Solution}

Our numerical algorithm is based on the semi-implicit finite difference scheme given in [20] for the isothermal viscoelastic case. As in [21, 28-31], we extend the algorithm to the temperature equation and take the implicit terms at the intermediate time level $(N+\xi)$, where $0 \leq \xi \leq 1$. The algorithm employed in [21] uses $\xi=1 / 2$; we will, however, follow the formulation in $[20,28-31]$ and thus take $\xi=1$ in this paper so that we can use larger time steps. The discretization of the governing equations is based on a linear Cartesian mesh and uniform grid on which finite differences are taken. We approximate both the second and first spatial derivatives with second-order central differences. The equations corresponding to the first and last grid point 


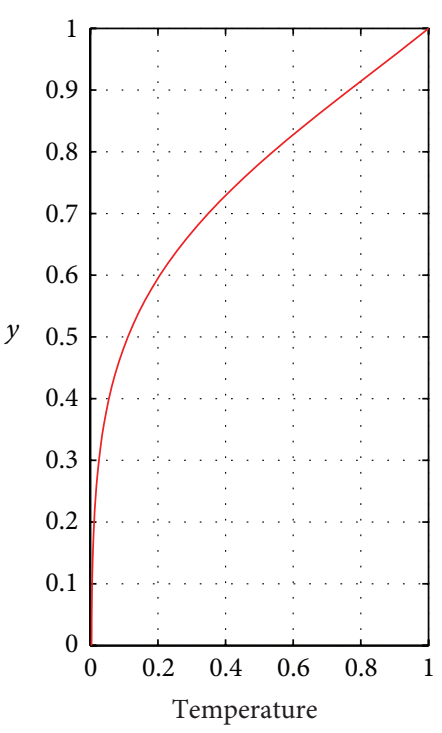

(a)

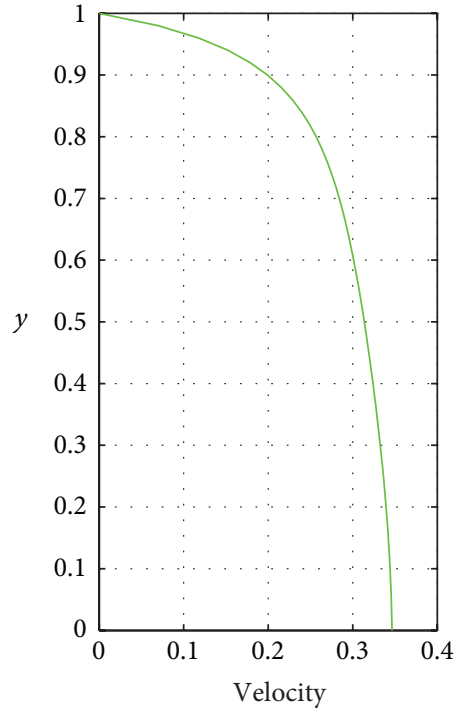

(b)

Figure 2: Velocity and temperature profiles at $t=0.5$.

are modified to incorporate the boundary conditions. The semi-implicit scheme for the velocity component reads

$$
\begin{aligned}
\operatorname{Re} & \frac{\left(w^{(N+1)}-w^{(N)}\right)}{\Delta t} \\
= & \left(a-b y^{n}\right) w_{y y}^{(N+\xi)}-M w^{(N+\xi)} \\
& +L+\operatorname{Gr} \theta^{(N)}+\frac{1}{y}\left[a-b(n+1) y^{n}\right] w_{y}^{(N)} .
\end{aligned}
$$

The equation for $w^{(N+1)}$ then becomes

$$
\begin{gathered}
-r_{1} w_{j-1}^{(N+1)}+\left(\operatorname{Re}+2 r_{1}+M \Delta t\right) w_{j}^{(N+1)} \\
-r_{1} w_{j+1}^{(N+1)}=\text { explicit terms, }
\end{gathered}
$$

where $r_{1}=\left(a-b y^{n}\right) \Delta t /\left(\Delta y^{2}\right)$. The solution procedure for $w^{(N+1)}$ thus reduces to inversion of tridiagonal matrices which is an advantage over a full implicit scheme. The semiimplicit integration scheme for the temperature equation is similar to that for the velocity component. Unmixed second partial derivatives of the temperature are treated implicitly:

$$
\begin{aligned}
\operatorname{Re} \operatorname{Pr} \frac{\theta^{(N+1)}-\theta^{(N)}}{\Delta t}= & \theta_{y y}^{(N+\xi)}+\frac{1}{y} \theta_{y}^{(N)} \\
& +\operatorname{Ra}\left[\left(\theta^{(N)}+h\right)^{4}-h^{4}\right] \\
& +\operatorname{Br}\left(a-b y^{n}\right)\left(w_{y}^{2}\right)^{(N)} .
\end{aligned}
$$

The equation for $\theta^{(N+1)}$ thus becomes

$$
-r \theta_{j-1}^{(N+1)}+(\operatorname{Re} \operatorname{Pr}+2 r) \theta_{j}^{(N+1)}-r \theta_{j+1}^{(N+1)}=\text { explicit terms, }
$$

where $r=\Delta t / \Delta y^{2}$. The solution procedure again reduces to inversion of tridiagonal matrices. The schemes (11) and (13) were checked for consistency. For $\xi=1$, these are first order accurate in time but second order in space. The schemes in [21] have $\xi=1 / 2$ which improves the accuracy in time to second order. We use $\xi=1$ here so that we are free to choose larger time steps and still converge to the steady solutions. The algorithm was also tested for both spatial and temporal convergence and shown to be independent of both mesh size and time step size.

\section{Results and Discussion}

Unless otherwise stated, we employ the parameter values: $\mathrm{Gr}=1, \operatorname{Pr}=25, \operatorname{Re}=0.5, a=1, b=0.7, n=3, m=0.2$, $M=0.5, \mathrm{Ra}=0.1, L=1, h=0.2, \mathrm{Br}=0.1, \Delta t=0.001$, and $t=50$.

4.1. General Profiles of Flow Quantities. In the early stage of thermal therapy treatment (say $t=0.5$ ), it is interesting to note that the blood velocity profile in a large vessel converges to a standard parabolic profile much earlier than does the temperature profile as illustrated in Figure 2. This is because large vessels exhibit large temperature gradients than smaller vessels. As such, the dissipation of heat from heated tissues, which is carried out by convection and conduction, is greater for large vessels due to a strong cooling effect. Consequently, the cooling effect of the blood flow in large vessels may result in underdosage of select tumor parts, since part of the surrounding tumor may not reach the desired treatment temperature. However, at much later time of thermal dosage administration (say $t=50$ ), the blood temperature profile increases transversely towards the arterial centerline in the same manner as the velocity profile (see Figure 3 ). This 


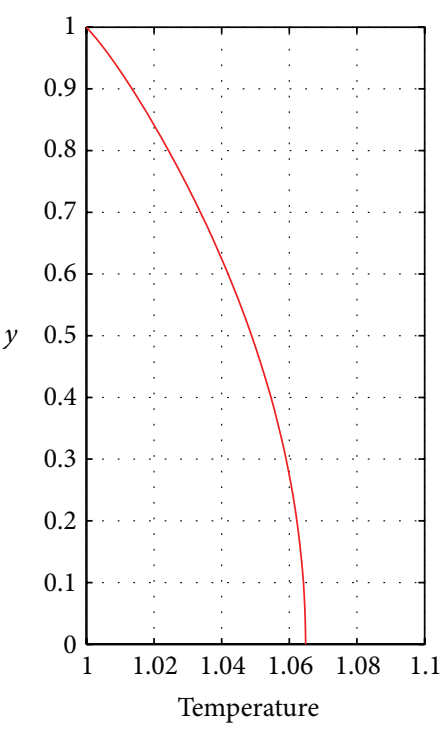

(a)

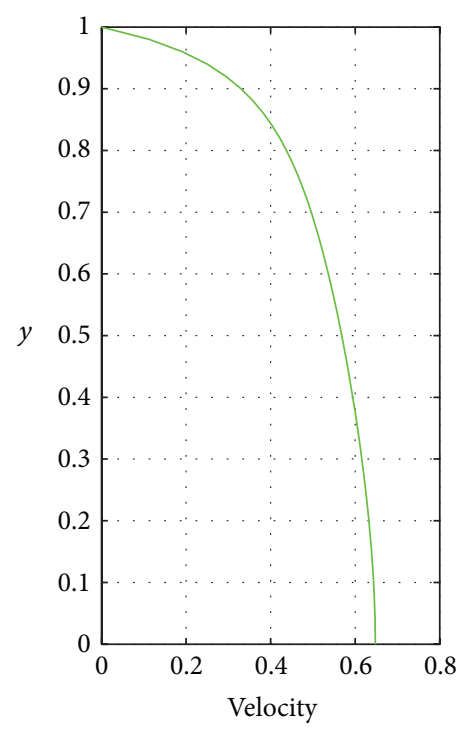

(b)

FIGURE 3: Velocity and temperature profiles at convergence; $t=50$.

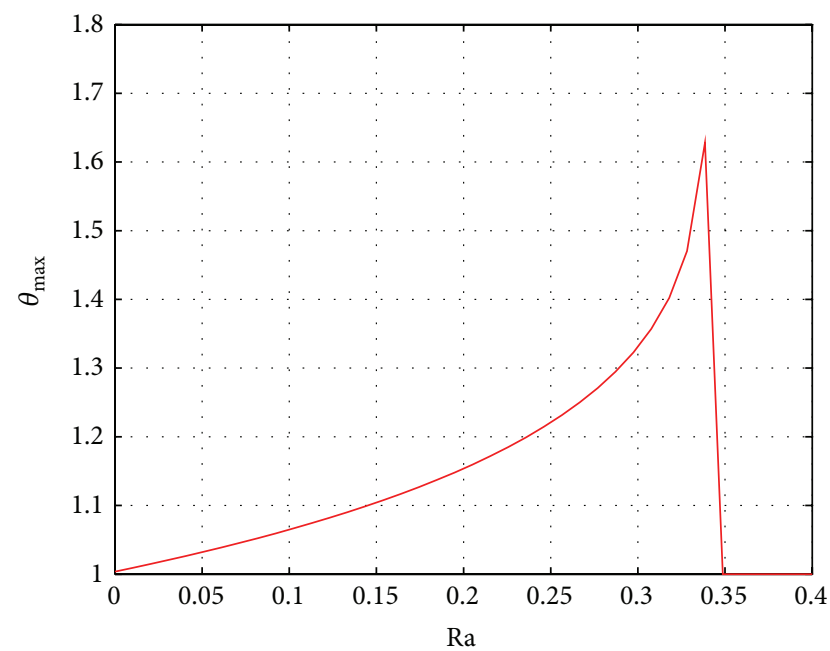

FIGURE 4: Blowup of fluid temperature; $t=50$.

enables the surrounding tumor parts to attain the desired treatment temperature.

We need to point out early on that the parameter Ra will need to be carefully controlled. Physically, the thermal radiation therapy is administered on the patient for time $t>0$. However, the therapy dose needs to be controlled in order to prevent development of hot spots (represented by blowup) in the flow system; see Figure 4 which plots the steady state maximum blood temperature against each value of Ra. Occurrence of such blowup phenomena may severely damage normal body tissue.

Significantly large dosage of radiation therapy (i.e., large $\mathrm{Ra}$ ) would lead to finite time temperature blowup since the terms associated with $\mathrm{Ra}$ are strong heat sources as with, say, the Frank-Kamenetskii parameter in reacting flows [21]. At our current material values, we notice that such blowup of solutions occurs below, say, $\mathrm{Ra}=0.35$. Our choices of $\mathrm{Ra}$ in this paper will thus be informed by Figure 4 .

4.2. Transient Solutions. As an immediate followup to the results of Figures 2 and 3, we display the transient solutions in Figure 5. As expected, Figure 5 shows that the blood velocity increases with time during the thermal therapy treatment.

As explained earlier, Figure 5 shows a progressive increase of arterial blood temperature with increasing time, until a steady state is reached which depicts an expected parabolic profile. The time evolution of the velocity and temperature with varying values of the radiation parameter along the arterial centerline is illustrated in Figure 6.

Since, as mentioned earlier, the radiation acts as a heat source within the blood, the arterial blood temperature (and hence also that along the centerline) should gradually increase with increasing radiation dosage; this is well illustrated in Figure 6. The resultant increase in temperature correspondingly increases the Boussinesq source terms in the momentum equation and hence also increases the blood velocity. This increase of velocity with radiation dosage is illustrated in Figure 6.

\subsection{Velocity Dependence on Magnetic Field and Hematocrit} Shape. The influences of the magnetic field and hematocrit shape on the velocity profile are shown in Figures 7 and 8, respectively.

Increasing the damping magnetic force predictably decreases the blood velocity as alluded to earlier and illustrated in Figure 7. A change in the hematocrit shape from the $n=2$ profile also decreases the velocity. This is expected since higher values of $n$ correspond to increased blood viscosity and hence reduced velocity. 


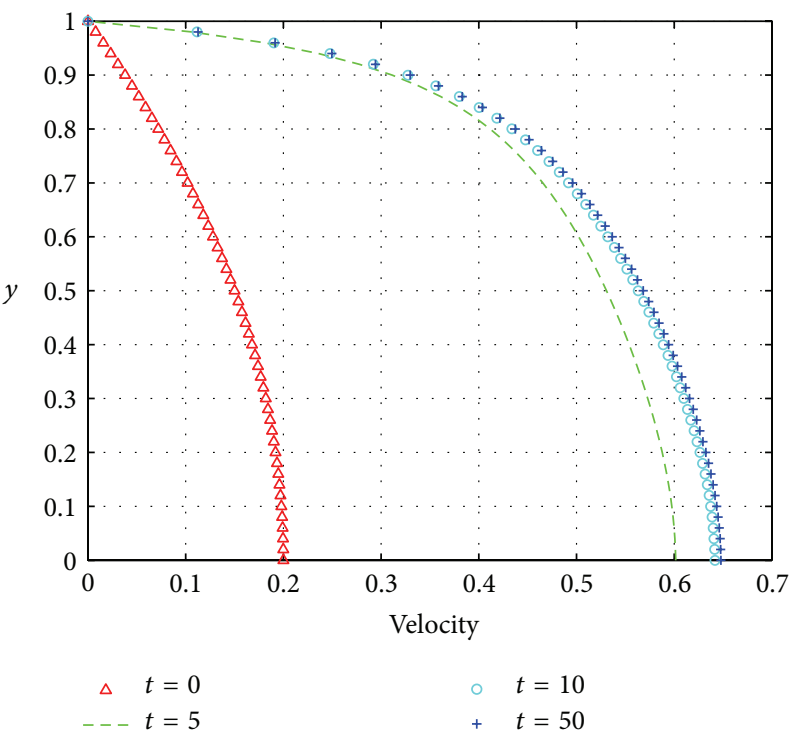

(a)

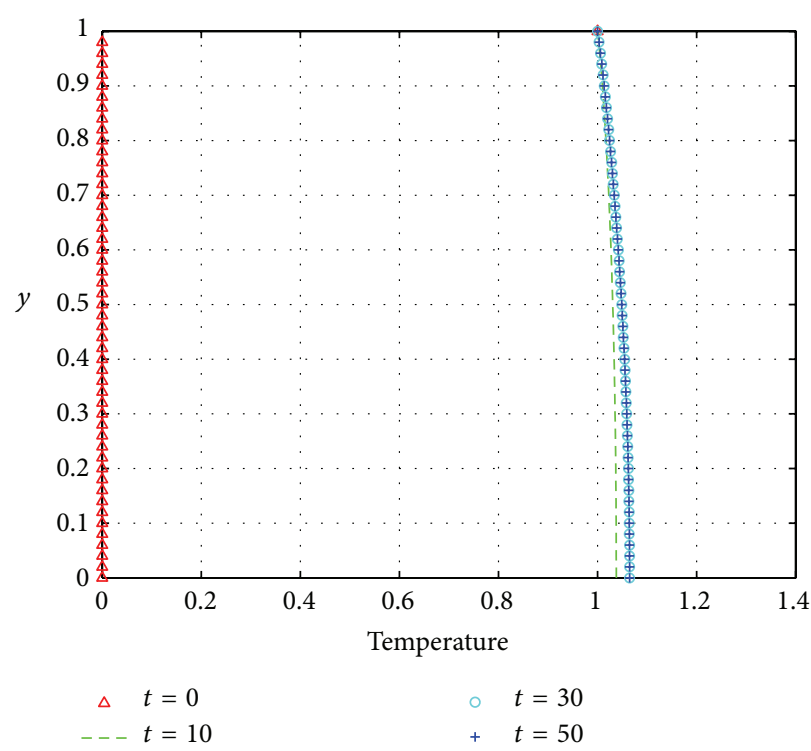

(b)

FIGURE 5: Developing velocity and temperature profiles.

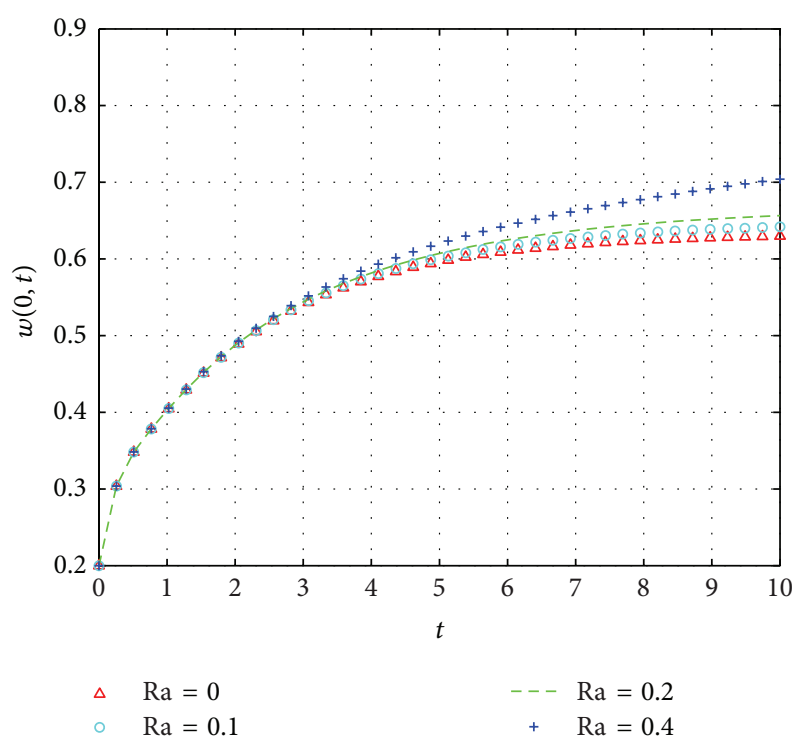

(a)

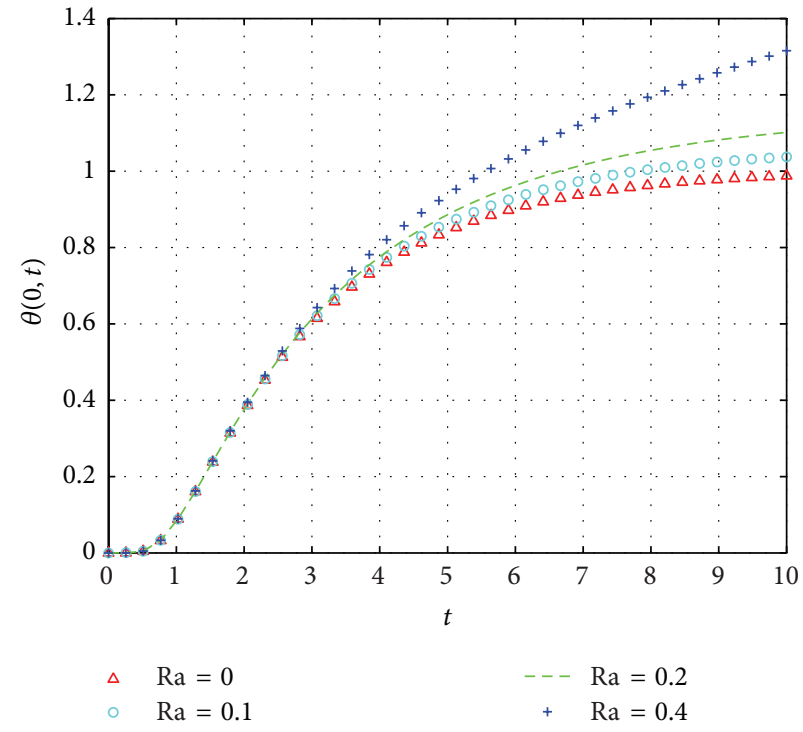

(b)

FIgURE 6: Evolution of velocity and temperature along $y=0$.

4.4. Wall Shear Stress and Wall Heat Transfer. The wall shear stress dependence on hematocrit shape is illustrated in Figure 9 for varying values of the radiation parameter. Similarly, Figure 10 shows the wall shear stress dependence on the magnetic field for varying values of $\mathrm{Gr}$.

Since increased values of $n$ correspond to increased viscosities and thus reduced velocities, the magnitude of the slope of the velocity correspondingly decreases (with increasing $n$ ) and hence also the wall shear stress, Figure 9. Increases in the thermal radiation dosage would necessarily also increase the blood temperature as mentioned earlier.
Thus, for fixed $n$, increasing the radiation dosage ( $\mathrm{Ra}$ ) would indirectly increase the blood velocity and hence also the wall shear stress as illustrated in Figure 9. This is so since the increase in $\mathrm{Ra}$ increases the blood temperature and hence also the source terms in the momentum equation.

The velocity profiles have been shown to decrease with increasing magnetic field force, $M$. This in turn means that the magnitude of the slope of the velocity near the wall would also decrease with increasing $M$, hence explaining the observed decrease in wall shear stress with increasing magnetic field force in Figure 10. At each value of the 


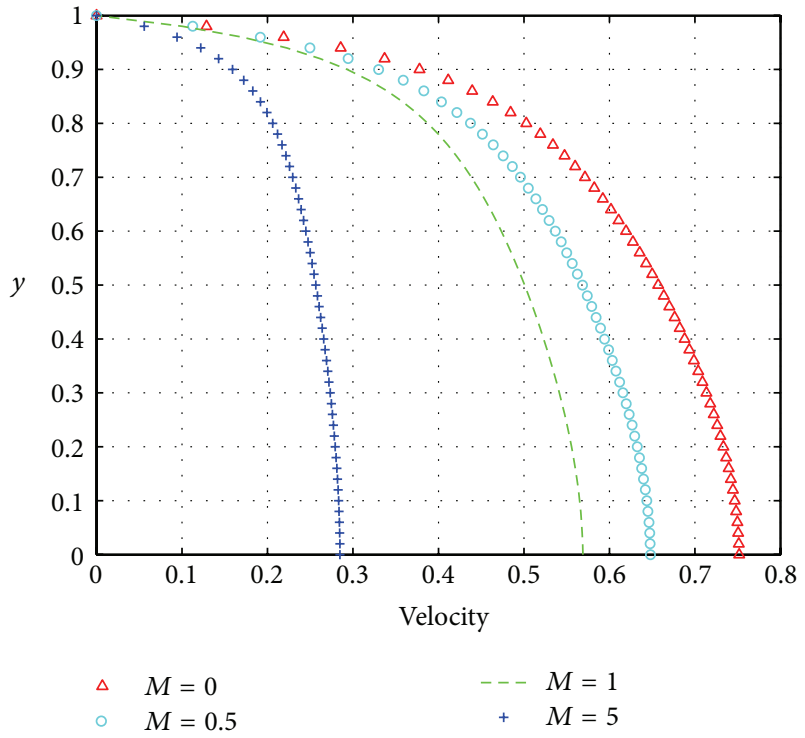

FIGURE 7: Effects of magnetic field on velocity; $t=50$.

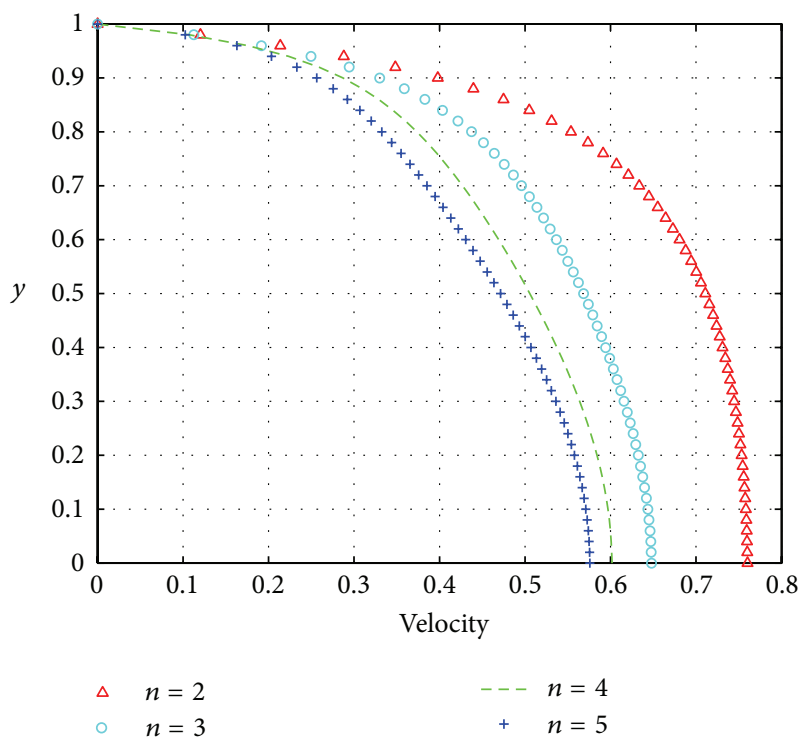

FIGURE 8: Effects of hematocrit shape on velocity; $t=50$.

magnetic field, an increase in the Grashof number would directly increase the velocity via the increased Boussinesq source terms and hence also the magnitude of the slope of the velocity at the wall.

The variation of the wall heat transfer with hematocrit shape for varying radiation parameter values is shown in Figure 11 and is similarly explained as with Figure 9. In particular, the velocity (and hence also the magnitude of the velocity gradient at the wall) decreases with increasing $n$. This in turn leads to a decrease in the viscous heating terms in the temperature equation leading to reduced blood temperatures. The decrease in the blood temperature (and hence also the magnitude of the temperature gradient at the wall) then leads to the corresponding decrease in wall heat

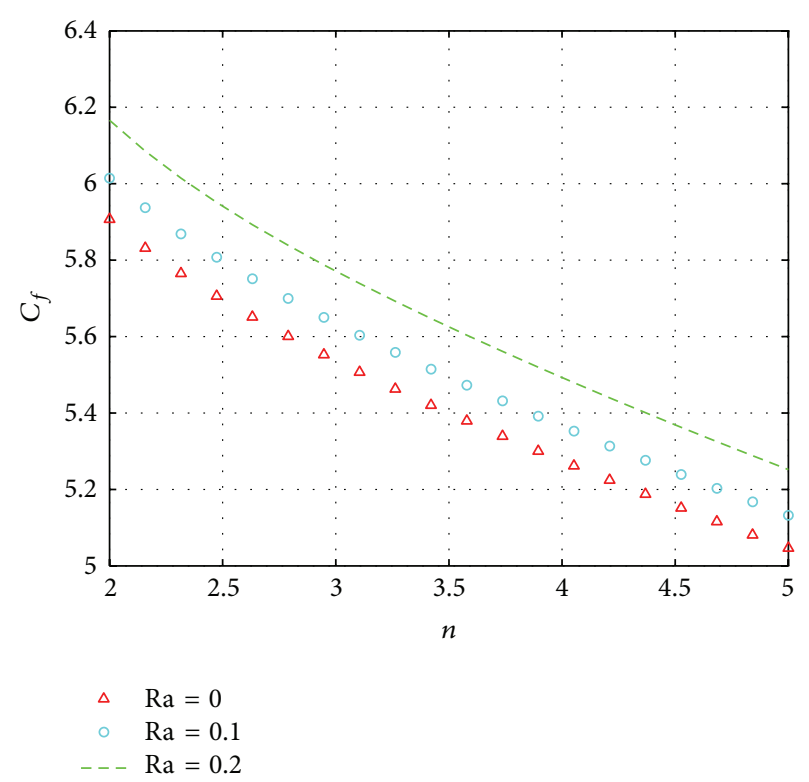

FIGURE 9: Variation of wall shear stress with hematocrit shape; $t=$ 50 .

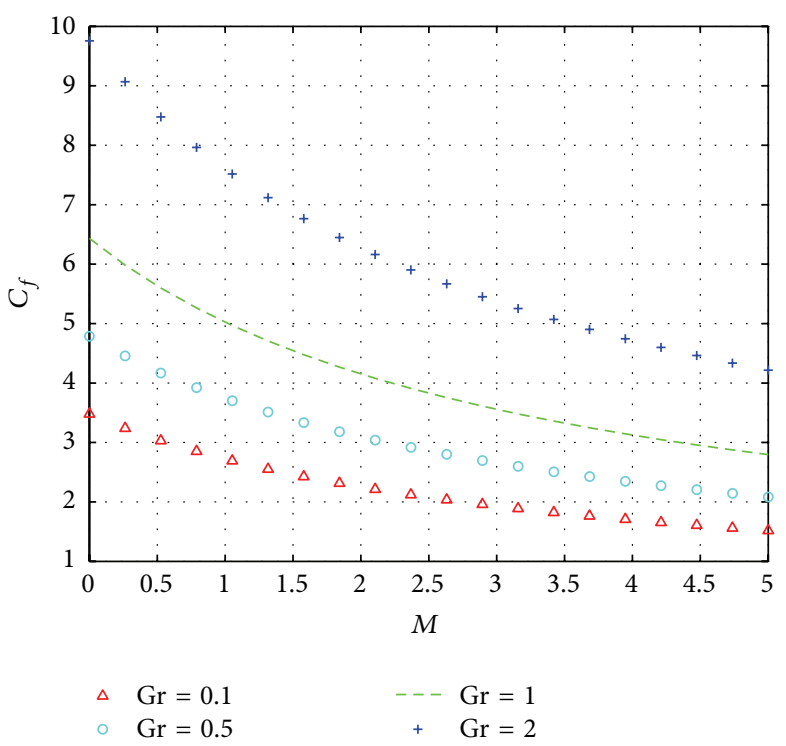

FIGURE 10: Variation of all shear stress magnetic field; $t=50$.

transfer (with increasing $n$ ) observed in Figure 11. At fixed $n$, increases in Ra directly lead to corresponding increases in the blood temperature as already noted. The increase in blood temperature (and hence also the magnitude of the temperature gradient at the wall) then leads to the corresponding increase in wall heat transfer (with increasing $\mathrm{Ra})$ observed in Figure 11.

The variation of the wall heat transfer with Brinkman number for varying values of the Grashof number is shown in Figure 12. Increases in the Brinkman number would correspondingly increase the magnitude of the viscous heating terms and thus also increase the arterial blood temperature. 


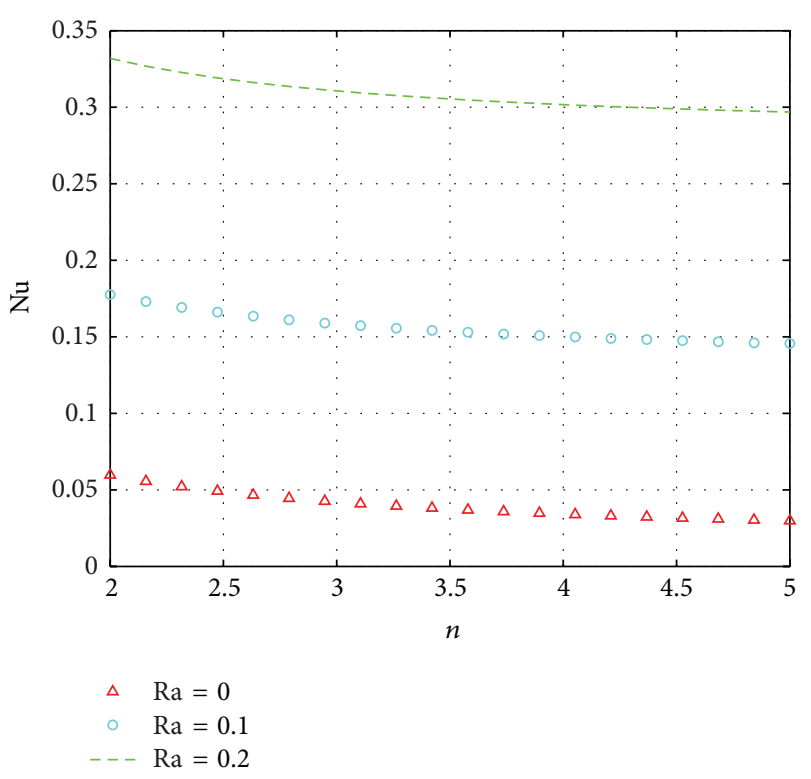

FIGURE 11: Variation of wall heat transfer with hematocrit shape; $t=$ 50.

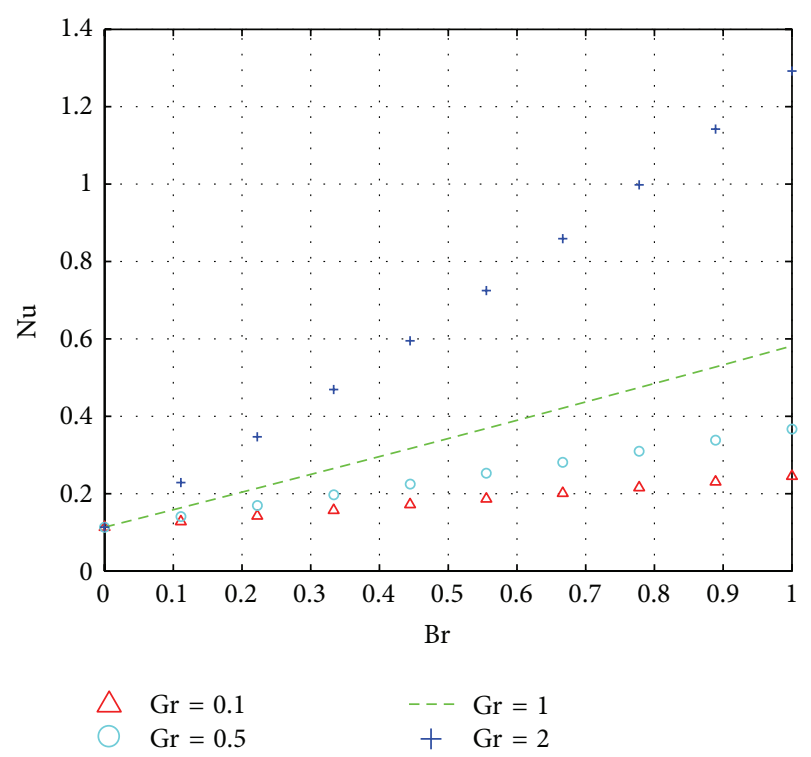

FIGURE 12: Variation of wall heat transfer with Brinkman number; $t=50$.

This leads to increases in the magnitudes of the temperature gradients at the wall and hence the observed increases in the wall heat transfer; see Figure 12. For each fixed Brinkman number, we again refer to the increase in the velocity with increasing Grashof number. This in turn similarly increases the magnitude of the viscous heating terms for each fixed value of the Brinkman number leading to the increased wall heat transfer shown in Figure 12.

\section{Conclusion}

We computationally investigate the influence of magnetic field force and thermal radiation on the dynamics of arterial blood with transversely varying viscosity and subjected to axisymmetric one-dimensional pressure driven flow under Boussinesq approximations. We observe that there is a transient increase in the blood velocity with an increase in the radiation absorption. Such increases in blood velocity and hence wall shear stress together with the possible development of hot spots in the flow system mean that the therapy dose needs to be carefully controlled. We also note that the blood velocity decreases with an increase in magnetic field intensity.

\section{Conflict of Interests}

The authors declare that there is no conflict of interests.

\section{References}

[1] A. Ogulu and A. R. Bestman, "Deep heat muscle treatment a mathematical model-I," Acta Physica Hungarica, vol. 73, no. 1, pp. 3-16, 1993.

[2] A. Ogulu and A. R. Bestman, "Deep heat muscle treatment a mathematical model-II," Acta Physica Hungarica, vol. 73, no. 1, pp. 17-27, 1993.

[3] T.-C. Shih, H.-L. Liu, and A. T.-L. Horng, "Cooling effect of thermally significant blood vessels in perfused tumor tissue during thermal therapy," International Communications in Heat and Mass Transfer, vol. 33, no. 2, pp. 135-141, 2006.

[4] S. A. Sapareto and W. C. Dewey, "Thermal dose determination in cancer therapy," International Journal of Radiation Oncology Biology Physics, vol. 10, no. 6, pp. 787-800, 1984.

[5] C. Damianou and K. Hynynen, "Focal spacing and near-field heating during pulsed high temperature ultrasound therapy," Ultrasound in Medicine and Biology, vol. 19, no. 9, pp. 777-787, 1993.

[6] J. van der Zee, "Heating the patient: a promising approach?" Annals of Oncology, vol. 13, no. 8, pp. 1173-1184, 2002.

[7] W. Levin, M. D. Sherar, B. Cooper, R. P. Hill, J. W. Hunt, and F.-F. Liu, "Effect of vascular occlusion on tumour temperatures during superficial hyperthermia," International Journal of Hyperthermia, vol. 10, no. 4, pp. 495-505, 1994.

[8] R. B. Roemer, "The local tissue cooling coefficient: a unified approach to thermal washout and steady-state perfusion calculations," International Journal of Hyperthermia, vol. 6, no. 2, pp. 421-430, 1990.

[9] H. H. Pennes, "Analysis of tissue and arterial blood temperatures in the resting human forearm," Journal of Applied Physiology, vol. 1, no. 2, pp. 93-122, 1948.

[10] O. I. Craciunescu and S. T. Clegg, "Pulsatile blood flow effects on temperature distribution and heat transfer in rigid vessels," Journal of Biomechanical Engineering, vol. 123, no. 5, pp. 500505, 2001.

[11] R. Siegel and M. Perlmutter, "Heat transfer for pulsating laminar duct flow," Journal of Heat Transfer, vol. 8, pp. 111-116, 1962.

[12] J. C. Chato, "Heat transfer to blood vessels," Journal of Biomechanical Engineering, vol. 102, no. 2, pp. 110-118, 1980.

[13] T. C. Shih, H. S. Kou, and W. L. Lin, "Effect of effective tissue conductivity on thermal dose distributions of living tissue with 
directional blood flow during thermal therapy,' International Communications in Heat and Mass Transfer, vol. 29, no. 1, pp. 115-126, 2002.

[14] M. C. Kolios, A. E. Worthington, D. W. Holdsworth, M. D. Sherar, and J. W. Hunt, "An investigation of the flow dependence of temperature gradients near large vessels during steady state and transient tissue heating," Physics in Medicine and Biology, vol. 44, no. 6, pp. 1479-1497, 1999.

[15] J. Creeze and J. J. W. Lagendijk, "Temperature uniformity during hyperthermia: the impact of large vessels," Physics in Medicine and Biology, vol. 37, no. 6, pp. 1321-1337, 1992.

[16] H. W. Cho and J. M. Hyun, "Numerical solutions of pulsating flow and heat transfer characteristics in a pipe," International Journal of Heat and Fluid Flow, vol. 11, no. 4, pp. 321-330, 1990.

[17] S. Y. K. Seo Young Kim, B. H. K. Byung Ha Kang, and J. M. H. Jae Min Hyun, "Heat transfer in the thermally developing region of a pulsating channel flow," International Journal of Heat and Mass Transfer, vol. 36, no. 17, pp. 4257-4266, 1993.

[18] J. Prakash, O. D. Makinde, and A. Ogulu, "Magnetic effect on oscillatory blood flow in a constricted tube," Botswana Journal of Technology, vol. 13, no. 1, pp. 45-50, 2004.

[19] O. D. Makinde, "Analysis of non-newtonian reactive flow in a cylindrical pipe," Journal of Applied Mechanics, Transactions ASME, vol. 76, no. 3, pp. 1-5, 2009.

[20] T. Chinyoka, Y. Y. Renardy, M. Renardy, and D. B. Khismatullin, "Two-dimensional study of drop deformation under simple shear for Oldroyd-B liquids," Journal of Non-Newtonian Fluid Mechanics, vol. 130, no. 1, pp. 45-56, 2005.

[21] T. Chinyoka, "Computational dynamics of a thermally decomposable viscoelastic lubricant under shear," Journal of Fluids Engineering, Transactions of the ASME, vol. 130, no. 12, Article ID 121201, 7 pages, 2008.

[22] T. J. Pedley, Fluid Dynamics of Large Blood Vessel, Cambridge Press, Cambridge, UK, 1980.

[23] L. Pauling and C. D. Coryell, "The magnetic properties and structure of hemoglobin, oxyhemoglobin and carbonmonoxyhemoglobin," Proceedings of the National Academy of Sciences of the United States of America, vol. 22, pp. 210-216, 1936.

[24] A. R. Rao and K. S. Deshikachar, "Physiological-type flow in a circular pipe in the presence of a transverse magnetic field," The Journal of the Indian Institute of Science, vol. 68, pp. 247-260, 1988.

[25] K. Haldar and S. N. Ghosh, "Effect of a magnetic field on blood flow through an indented tube in the presence of erythrocytes," Indian Journal of Pure and Applied Mathematics, vol. 25, no. 3, pp. 345-352, 1994.

[26] P. Cheng, "Two dimensional radiating gas flow by a moment method," The American Institute of Aeronautics and Astronautics, vol. 2, pp. 1662-1664, 1964.

[27] M. M. Lih, Transport Phenomenon in Medicine and Biology, John Wiley and Sons, New York, NY, USA, 1969.

[28] T. Chinyoka, "Poiseuille flow of reactive Phan-Thien-Tanner liquids in 1D channel flow," Journal of Heat Transfer, vol. 132, no. 11, Article ID 111701, 2010.

[29] T. Chinyoka, "Suction-injection control of shear banding in non-isothermal and exothermic channel flow of JohnsonSegalman liquids," Journal of Fluids Engineering, Transactions of the ASME, vol. 133, no. 7, Article ID 071205, 2011.

[30] I. E. Ireka and T. Chinyoka, "Non-isothermal flow of a JohnsonSegalman liquid in a lubricated pipe with wall slip," Journal of Non-Newtonian Fluid Mechanics, vol. 192, pp. 20-28, 2013.
[31] T. Chinyoka, S. Goqo, and B. I. Olajuwon, "Computational analysis of gravity driven flow of a variable viscosity viscoelastic fluid down an inclined plane," Computers \& Fluids, vol. 84, no. 15, pp. 315-326, 2013. 


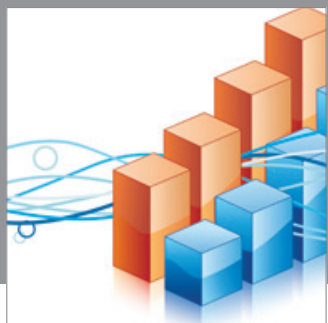

Advances in

Operations Research

mansans

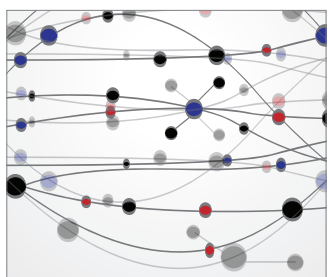

The Scientific World Journal
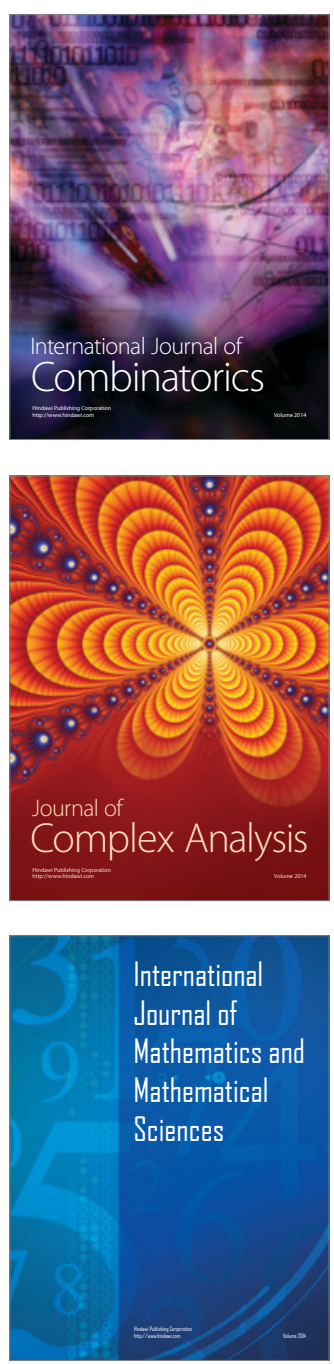
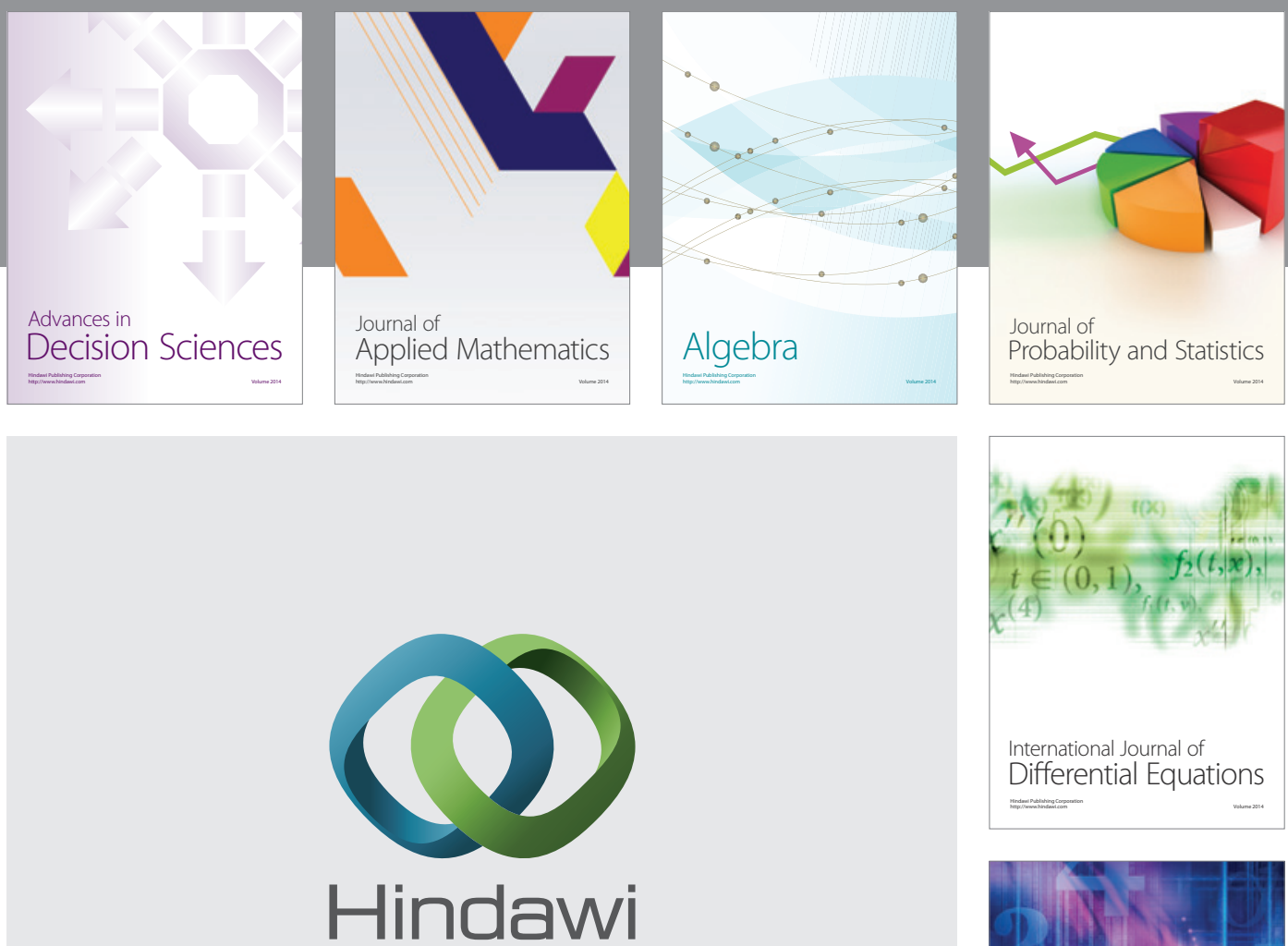

Submit your manuscripts at http://www.hindawi.com
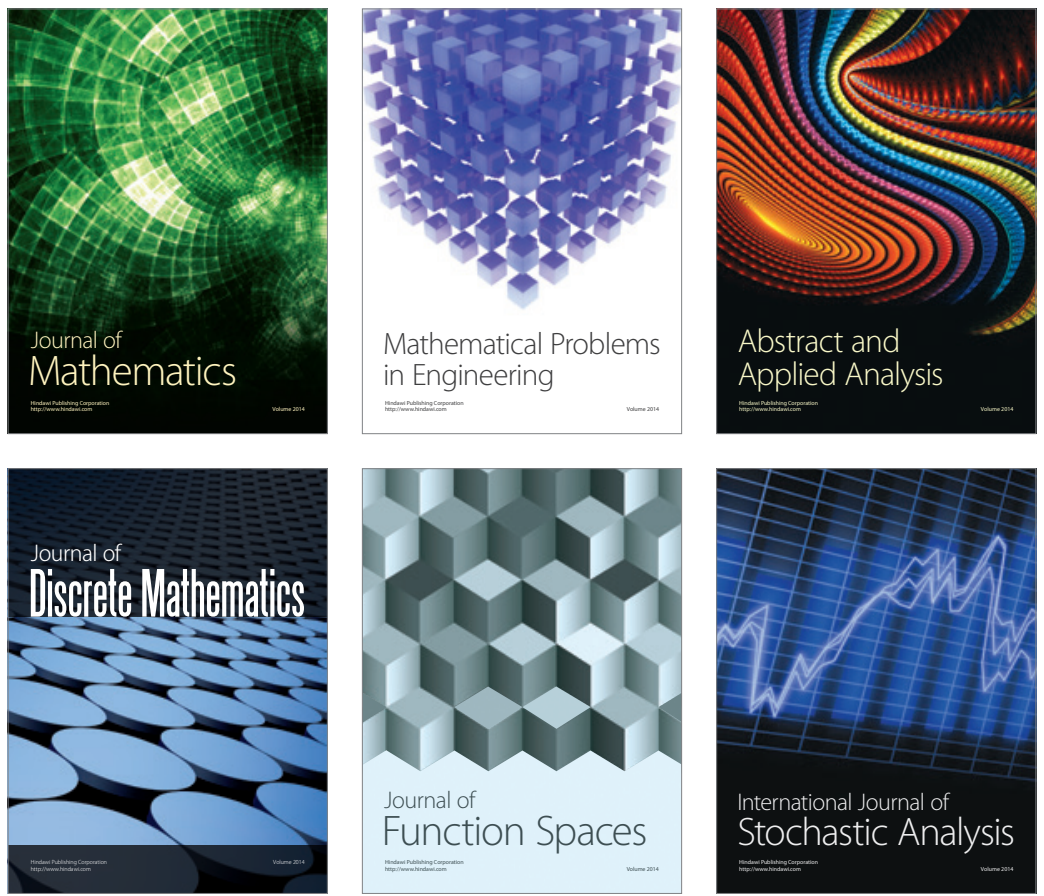

Journal of

Function Spaces

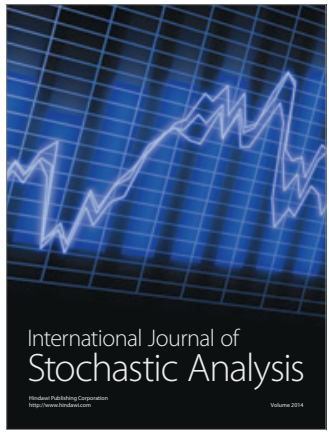

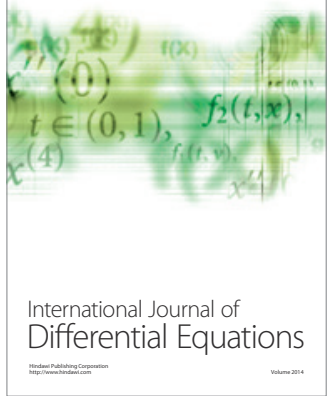
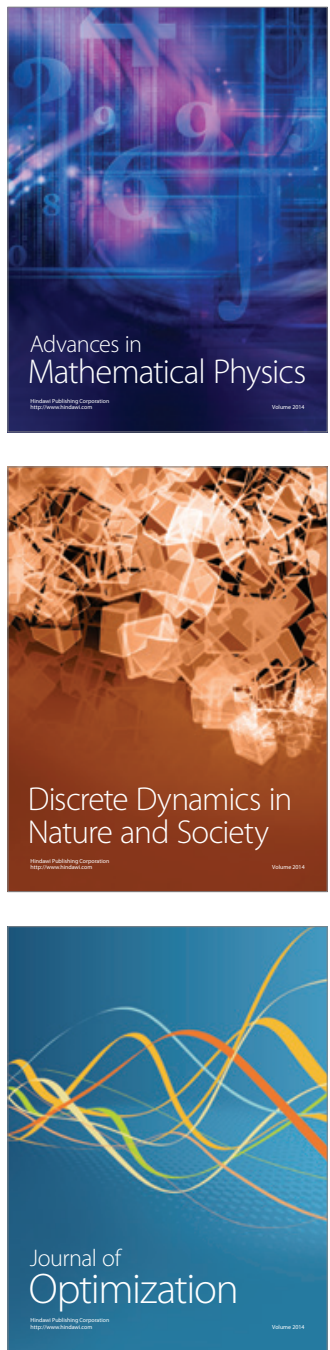